\title{
Holographic entropy bound in higher-dimensional spacetimes
}

\author{
Shahar Hod \\ The Ruppin Academic Center, Emeq Hefer 40250, Israel \\ and The Hadassah Academic College, Jerusalem 91010, Israel
}

(Received 8 February 2018; published 20 June 2018)

\begin{abstract}
The celebrated holographic entropy bound asserts that, within the framework of a self-consistent quantum theory of gravity, the maximal entropy (information) content of a physical system is given by one quarter of its circumscribing area: $S \leq S_{\max }=\mathcal{A} / 4 \ell_{P}^{2}$ (here $\ell_{P}$ is the Planck length). An intriguing possible counterexample to this fundamental entropy bound, which involves homogenous weakly self-gravitating confined thermal fields in higher-dimensional spacetimes, was proposed almost a decade ago. Interestingly, in the present paper we shall prove that this composed physical system, which at first sight seems to violate the holographic entropy bound, actually conforms to the entropy-area inequality $S \leq \mathcal{A} / 4 \ell_{P}^{2}$. In particular, we shall explicitly show that the homogeneity property of the confined thermal fields sets an upper bound on the entropy content of the system. The present analysis therefore resolves the apparent violation of the holographic entropy bound by confined thermal fields in higher-dimensional spacetimes.
\end{abstract}

DOI: 10.1103/PhysRevD.97.126012

\section{INTRODUCTION}

The holographic principle [1-3] provides a remarkably simple geometric answer to the following physically interesting question: what is the maximal entropy (information) content of a spatially bounded physical system? In particular, the holographic entropy bound asserts that the remarkably compact inequality [1-5]

$$
S \leq S_{\max }=\frac{\mathcal{A}}{4 \ell_{P}^{2}}
$$

sets a fundamental upper bound on the entropy content of a physical system of circumference area $\mathcal{A}$ [6-9]. Interestingly, the inequality (1) is saturated by stationary black holes in generic $(D+1)$-dimensional spacetimes. This fact suggests that, for a given surface area $\mathcal{A}$, these fundamental solutions of the Einstein field equations are characterized by the maximally allowed entropy: $S_{\mathrm{BH}}=S_{\max }(\mathcal{A})$.

It is widely believed $[1-3,10]$ that the holographic entropy bound (1) reflects a fundamental physical feature of the elusive quantum theory of gravity. Interestingly, the bound is known to be valid for $(3+1)$-dimensional isolated physical systems with negligible self-gravity [3]. One naturally wonders whether the holographic entropy bound (1) is also respected by weakly self-gravitating physical systems in higher-dimensional spacetimes [11,12].

Published by the American Physical Society under the terms of the Creative Commons Attribution 4.0 International license. Further distribution of this work must maintain attribution to the author(s) and the published article's title, journal citation, and DOI. Funded by SCOAP ${ }^{3}$.
This is a highly nontrivial question since one expects the entropy content of a physical system to be an increasing function of the number $D$ of spatial dimensions. In particular, for a $(D+1)$-dimensional physical system with a fixed amount of energy $E$, the larger is $D$, the more distinct ways there are to populate the discrete energy levels of the physical system $[11,12]$. It is therefore of physical interest to test the validity of the holographic entropy bound (1) in higher-dimensional spacetimes.

The main goal of the present paper is to calculate the entropy content of bounded homogenous thermal fields in generic $(D+1)$-dimensional spacetimes and to test the validity of the fundamental holographic entropy bound (1) in these higher-dimensional spacetimes.

\section{ENTROPY CONTENT OF $(D+1)$-DIMENSIONAL BOUNDED THERMAL FIELDS}

In a highly interesting paper, Bekenstein [11] has calculated the entropy content of a $(D+1)$-dimensional physical system which is composed of massless homogenous thermal fields of total energy $E$ which are bounded inside a spherical box of proper radius $R$. As explicitly shown in Ref. [11], the thermal entropy content of this physical system is given by the functional relation

$$
S=C_{D}(1+1 / D) N^{\frac{1}{D+1}}(R E / \hbar)^{\frac{D}{D+1}}
$$

where

$$
C_{D} \equiv\left[\frac{2 \zeta(D+1) \Gamma\left(\frac{D+1}{2}\right)}{\pi^{1 / 2} \Gamma\left(\frac{D}{2}\right)}\right]^{\frac{1}{D+1}}
$$


and the dimensionless parameter $N$ is the effective number of polarization states (degrees of freedom) of the higher-dimensional thermal fields: a massless scalar field contributes 1 to $N$, a massless fermionic field in a $(D+1)$ dimensional spacetime contributes $1-2^{-D}$ to $N$ [11], an electromagnetic field in a $(D+1)$-dimensional spacetime contributes $D-1$ to $N$ [13], and the graviton in a $(D+1)$ dimensional spacetime contributes $(D+1)(D-2) / 2$ to $N$ [13]. It is important to emphasize the fact that the entropy formula (2) was derived in Ref. [11] for the specific case of homogenous massless fields in thermal equilibrium. [The homogeneity assumption made in Ref. [11] in deriving the entropy formula (2) corresponds to the assumption (19) made below.]

As discussed in Refs. [11,12], the compact analytical expression (2) derived in Ref. [11] for the entropy content of the $(D+1)$-dimensional thermal fields is valid under the following two physical assumptions: 1) The bounded physical system is weakly self-gravitating. As explicitly shown in Ref. [12], this physical condition can be characterized by the dimensionless strong inequality

$$
\eta \equiv \frac{16 \pi R E}{(D-1) \mathcal{A}} \ll 1,
$$

where $\mathcal{A}$ is the $(D-1)$-dimensional surface area of the bounded system. 2) In addition, the analytical expression (2) for the entropy content of the bounded fields is only valid in the thermodynamic regime. As discussed in Refs. [11,12], the thermodynamic (continuum) treatment of the confined fields is valid provided many field quanta are thermally excited. In particular, as explicitly shown in Ref. [12], this physical condition can be characterized by the dimensionless strong inequality

$$
\xi \equiv \frac{D}{C_{D}}\left(\frac{N \hbar}{R E}\right)^{\frac{D}{D+1}} \ll 1 .
$$

Substituting Eqs. (4) and (5) into Eq. (2), one finds

$$
S=\left(\frac{C_{D}}{D}\right)^{\frac{D+1}{D}} \frac{D^{2}-1}{4 \pi} \eta \xi^{\frac{1}{D}} \cdot \frac{\mathcal{A}}{4 \hbar}
$$

for the thermal entropy of the bounded $(D+1)$-dimensional fields. Intriguingly, the entropy-area relation (6) suggests, at first sight, that in the large- $D$ regime $[12,14,15]$

$$
D>\frac{4 \pi}{\eta} \gg 1,
$$

the entropy content of this higher-dimensional physical system can violate the holographic entropy bound (1) [12]

As discussed above, the holographic entropy bound (1) is widely believed $[1-3,10]$ to reflect a fundamental physical property of the elusive quantum theory of gravity. Thus, for the advocates of the holographic principle, the task remains to find out how the holographic entropy bound (1) eventually survives the serious challenge which is imposed [see Eqs. (6) and (7)] by the higher-dimensional confined thermal fields.

The main goal of the present paper is to explore the (in) validity of the holographic entropy bound (1) in higherdimensional physical systems which are made of confined thermal fields. As we shall show in the next section, the physical assumption of homogeneity made in Refs. [11,12] sets an upper bound on the entropy content of the fields. In particular, below we shall explicitly prove that, properly taking into account the homogeneity property of the confined fields, one finds that the higher-dimensional thermal system actually respects the holographic entropy bound (1).

\section{THE HOMOGENEITY ASSUMPTION AND THE HOLOGRAPHIC ENTROPY BOUND}

In the present section we shall show that the homogeneity assumption of the confined fields, on which the entropy formula (2) of Ref. [11] is based, sets an upper bound on the dimensionless physical quantity $R E / \hbar$ which characterizes the spatially bounded $(D+1)$-dimensional physical system. In particular, we shall explicitly prove that, for higher-dimensional spacetimes, the new bound on the dimensionless quantity $R E / \hbar$, which stems from the homogeneity assumption [see Eq. (23) below], is stronger than the formerly used bound (4) which stems from the weak gravity assumption.

The line element of a curved $(D+1)$-dimensional spherically symmetric spacetime can be expressed in the form

$$
d s^{2}=e^{\nu} d t^{2}-e^{\lambda} d r^{2}-r^{2} d \Omega,
$$

where $\nu=\nu(r)$ and $\lambda=\lambda(r)$ are dimensionless metric functions and $d \Omega$ is the angular line element of a unit $(D-1)$-dimensional sphere. Assuming an isotropic matter distribution whose energy-momentum tensor is given by $T_{\nu}^{\mu}=\operatorname{diag}(\rho,-p,-p, \ldots,-p)$ [16], the $(D+1)$-dimensional Einstein field equations, $R_{\mu \nu}=8 \pi\left[T_{\mu \nu}-\frac{1}{D-1} g_{\mu \nu} T\right]$, take the form [17]

$$
e^{-\lambda}\left(\frac{\lambda^{\prime}}{r}-\frac{D-2}{r^{2}}\right)+\frac{D-2}{r^{2}}=\frac{16 \pi}{D-1} \rho,
$$

and

$$
e^{-\lambda}\left(\frac{\nu^{\prime}}{r}+\frac{D-2}{r^{2}}\right)-\frac{D-2}{r^{2}}=\frac{16 \pi}{D-1} p .
$$

The mass (energy) contained within a $D$-dimensional sphere of radius $r$ is given by $[18,19]$

$$
E(r)=A_{D-1} \int_{0}^{r} \rho\left(r^{\prime}\right) r^{\prime D-1} d r^{\prime},
$$

where 
TABLE I. The prefactor $\alpha(D)$ of the entropy-area relation (24). One finds that $\alpha(D)$ is a monotonically decreasing function of the number $D$ of spatial dimensions with the property $\max _{D}\{\alpha(D)\}=\alpha(D=3) \simeq 0.0819$. Taking cognizance of Eq. (24), one deduces that the $(D+1)$-dimensional homogenous thermal fields conform to the holographic entropy bound (1).

\begin{tabular}{lcccccccc}
\hline \hline$D$ & 3 & 4 & 5 & 6 & 7 & 8 & 9 & 10 \\
\hline$\alpha(D)$ & 0.0819 & 0.0471 & 0.0343 & 0.0274 & 0.0229 & 0.0197 & 0.0174 & 0.0156 \\
\hline \hline
\end{tabular}

$$
A_{D-1}=\frac{2 \pi^{D / 2}}{\Gamma(D / 2)}
$$

is the surface area of a unit $(D-1)$-dimensional sphere. From Eq. (9) one finds the simple relation [17]

$$
e^{-\lambda}=1-\frac{2 \bar{E}(r)}{r^{D-2}}
$$

where

$$
\bar{E}(r) \equiv \frac{8 \pi}{(D-1) A_{D-1}} E(r) .
$$

Substituting Eq. (13) into Eq. (10), one obtains the differential relation [17]

$$
\nu^{\prime}=\frac{2}{D-1} \cdot \frac{(D-1)(D-2) \bar{E}(r)+8 \pi p r^{D}}{r^{D-1}\left[1-2 \bar{E}(r) / r^{D-2}\right]} .
$$

In addition, substituting the Einstein field equations (9) and (10) into the conservation equation

$$
T_{r ; \mu}^{\mu}=0
$$

one finds the differential relation [17]

$$
\frac{d p}{d r}=-\frac{1}{2} \nu^{\prime}(\rho+p)
$$

for the gradient of the radial pressure. Taking cognizance of Eqs. (15) and (17), one obtains the generalized $[(D+1)$ dimensional] Tolman-Oppenheimer-Volkov equation

$$
\mathcal{F} \equiv \frac{r}{p} \cdot \frac{d p}{d r}=-\frac{(1+\rho / p)\left[(D-1)(D-2) \bar{E}(r)+8 \pi p r^{D}\right]}{(D-1) r^{D-2}\left[1-2 \bar{E}(r) / r^{D-2}\right]}
$$

At this point, it is important to emphasize again that the expression (2) for the entropy content of the higherdimensional physical system is valid for weakly selfgravitating confined thermal fields of spatially homogeneous (constant) energy density and pressure [11]. This homogeneity assumption made in Refs. $[11,12]$ corresponds to the dimensionless strong inequality [see Eq. (18)]

$$
\mathcal{F} \ll 1
$$

Taking cognizance of Eqs. (18) and (19), and using the relation [20]

$$
p=\rho / D
$$

for massless radiation fields in $D$ spatial dimensions, one finds the characteristic strong inequality [21]

$$
(D-2)(D+1) \frac{\bar{E}(R)}{R^{D-2}} \ll 1
$$

for the nearly homogenous entropy-bearing thermal fields. Substituting Eq. (14) into Eq. (21), one obtains the strong inequality

$$
\frac{8 \pi(D-2)(D+1)}{(D-1) A_{D-1}} \cdot \frac{E}{R^{D-2}} \ll 1
$$

which yields the characteristic small dimensionless ratio [22]

$$
\epsilon \equiv \frac{8 \pi(D-2)(D+1)}{D-1} \cdot \frac{R E}{\mathcal{A}} \ll 1
$$

for the nearly homogenous confined thermal fields.

Finally, substituting Eqs. (5) and (23) into Eq. (2), one obtains the functional expression

$$
S=\alpha(D) \cdot \frac{\xi^{\frac{1}{D}} \epsilon \mathcal{A}}{4 \hbar}
$$

for the entropy of the confined $(D+1)$-dimensional homogenous thermal fields, where

$$
\alpha(D) \equiv \frac{(D-1) C_{D}^{\frac{D+1}{D}}}{2 \pi(D-2) D^{\frac{D+1}{D}}}
$$

In Table I we present the numerically computed values of the $D$-dependent prefactor $\alpha(D)$. The numerically computed values of the prefactor reveal the fact that $\alpha(D)$ is a monotonically decreasing function of the number $D$ of spatial dimensions with the property $\max _{D}\{\alpha(D)\}=$ $\alpha(D=3) \simeq 0.0819$.

Interestingly, taking cognizance of the strong dimensionless inequalities (5) and (23) and the data presented in Table I, one deduces that, for generic values of the number $D$ of spatial dimensions, the entropy content (24) of the 
$(D+1)$-dimensional thermal fields actually conforms to the holographic entropy bound (1).

\section{SUMMARY}

The holographic entropy bound [1-3] asserts that the maximal entropy content of a bounded physical system is given (in Planck units) by one quarter of its circumscribing area: $S \leq \mathcal{A} / 4 \ell_{P}^{2}$ [see Eq. (1)]. It is widely believed [1-3,10] that this characteristic entropy bound reflects a fundamental physical property of the elusive quantum theory of gravity. It is therefore quite surprising that the entropy of homogenous weakly self-gravitating confined thermal fields in higherdimensional spacetimes seems, at first sight, to violate the entropy-area relation (1) [see Eqs. (6) and (7)] [12].

Interestingly, in the present paper we have explicitly proved that the homogeneity assumption made in Refs. [11,12] for the confined thermal fields [see Eq. (23)] sets an upper bound on the entropy content of the physical system. In particular, it has been shown that, properly taking into account the assumed homogeneity property of the spatially bounded physical system, one finds that the higher-dimensional thermal fields actually conform to the holographic entropy bound (1). Most importantly, from Eqs. (23) and (24) one obtains the asymptotic expression [14]

$$
S=\frac{\epsilon}{2 \pi D} \cdot \frac{\mathcal{A}}{4 \hbar} \ll \frac{\mathcal{A}}{4 \hbar} \quad \text { for } D \gg 1
$$

for the entropy content of the higher-dimensional fields in the large- $D$ regime. The present analysis therefore resolves the apparent violation [12] of the holographic entropy bound by confined thermal fields in higher-dimensional spacetimes.

\section{ACKNOWLEDGMENTS}

This research is supported by the Carmel Science Foundation. I thank Yael Oren, Arbel M. Ongo, Ayelet B. Lata, and Alona B. Tea for stimulating discussions.
[1] G. 't Hooft, in Salamfestschrift: A Collection of Talks from the Conference on Highlights of Particle and Condensed Matter Physics, ICTP, Trieste, Italy, 1993, edited by A. Aly, J. Ellis, and S. Randjbar-Daemi (World Scientific, Singapore 1993).

[2] L. Susskind, J. Math. Phys. (N.Y.) 36, 6377 (1995).

[3] J. D. Bekenstein, Phys. Lett. B 481, 339 (2000).

[4] We shall use natural units in which $G=c=k_{B}=1$.

[5] Here $\ell_{\mathrm{P}}=\sqrt{G \hbar / c^{3}}$ is the characteristic Planck length.

[6] See Refs. [7-9] for alternative entropy bounds.

[7] J. D. Bekenstein, Phys. Rev. D 23, 287 (1981).

[8] S. Hod, Phys. Rev. D 61, 024018 (1999).

[9] J. D. Bekenstein and A. E. Mayo, Phys. Rev. D 61, 024022 (1999); S. Hod, Phys. Rev. D 61, 024023 (1999).

[10] J. D. Bekenstein, Contemp. Phys. 45, 31 (2004); Found. Phys. 35, 1805 (2005).

[11] J. D. Bekenstein, Phys. Rev. D 49, 1912 (1994).

[12] S. Hod, Phys. Lett. B 695, 294 (2011); 700, 75 (2011).

[13] V. Cardoso, M. Cavaglia, and L. Gualtieri, J. High Energy Phys. 02 (2006) 021; H. Alnes, K. Olaussen, F. Ravndal, and I. K. Wehus, J. Phys. A 40, F315 (2007).
[14] Here we have used the asymptotic relations $C_{D} \rightarrow 1$, $D^{1+1 / D} \rightarrow D[1+(\ln D) / D]$, and $\xi^{\frac{1}{D}} \rightarrow 1$ for $D \gg 1$.

[15] The strong inequality on the rhs of Eq. (7) stems from the fact that $\eta \ll 1$ [see Eq. (4)].

[16] Here $\rho$ is the energy density and $p$ is the corresponding pressure component.

[17] J. P. de Leon and N. Cruz, Gen. Relativ. Gravit. 32, 1207 (2000).

[18] F. R. Tangherlini, Nuovo Cimento 27, 636 (1963).

[19] G. Kunstatter, Phys. Rev. Lett. 90, 161301 (2003).

[20] H. Alnes, F. Ravndal, and I. K. Wehus, J. Phys. A 40, 14309 (2007).

[21] Here we have used the relation $p>0$ which implies $(D-1)(D-2) \bar{E}(r)+8 \pi p r^{D}>(D-1)(D-2) \bar{E}(r)$. In addition, we have used here the inequality $1-$ $2 \bar{E}(r) / r^{D-2}<1$ for the spatially regular configurations. One therefore finds $|\mathcal{F}|>\frac{(1+\rho / p)(D-2) \bar{E}(r)}{r^{D-2}}$.

[22] Here we have used the relation $\mathcal{A}=A_{D-1} R^{D-1}$ for the $(D-1)$-dimensional surface area of the bounded spherical system. 